\title{
ELF magnetic field receiver: frequency performance and natural signals detection
}

Receptor ELF de campo magnético: desempeño en frecuencia y detección de señales naturales

\author{
L. M. Carducci ${ }^{*}$, R. Alonso ${ }^{\dagger}$ and W. G. Fano ${ }^{\dagger}$ \\ *Laboratorio de Procesamiento de Señales y Comunicaciones \\ ${ }^{\dagger}$ Laboratorio de Radiación Electromagnética \\ Facultad de Ingeniería, Universidad de Buenos Aires, Argentina \\ lcarducci@fi.uba.ar
}

Recibido: 28/06/21; Aceptado: 09/09/21

\begin{abstract}
This article presents the analog stage analysis of an extremely low frequency (ELF) magnetic field receiver. Details are described about the characterization and modeling of the antenna used, as well as the frequency behavior of the system. To achieve higher sensitivity, a coil was manufactured with a considerably high inductance $(800 \mathrm{H})$, in addition to a high-gain amplification stage $(88 \mathrm{~dB})$. An equivalent circuit model was defined for the antenna, and its parasitic elements were determined by laboratory measurements and computational approximations. The acquisition system has a digitization stage with a sampling frequency of $100 \mathrm{~Hz}$. To study its behavior against possible aliasing effects, an analysis of the analog stage in the frequency domain was carried out, determining the optimal operating conditions. Field tests of the equipment were carried out in the mountains of Villa Alpina, Cordoba, Argentina. Taking into account that the system was designed for the measurement and study of natural electromagnetic phenomena in the ELF band, the first six Schumann resonances have been successfully captured and detected.
\end{abstract}

Keywords: loop antenna; ELF; magnetic fields; acquisition system; Schumann resonance.

Resumen- En este artículo se presenta el análisis de la etapa analógica de un receptor de campo magnético para frecuencias extremadamente bajas (ELF). Se describen detalles sobre la caracterización y modelado de la antena utilizada, así como el comportamiento en frecuencia del sistema. Para lograr una mayor sensibilidad, se fabricó una bobina con una inductancia considerablemente alta $(800 \mathrm{H})$, además de una etapa de amplificación de alta ganancia $(88$ dB). Se definió un modelo de circuito equivalente para la antena y se determinaron sus elementos parásitos mediante mediciones de laboratorio y aproximaciones computacionales. El sistema de adquisición tiene una etapa de digitalización con una frecuencia de muestreo de $100 \mathrm{~Hz}$. Para estudiar su comportamiento frente a posibles efectos de aliasing, se realizó un análisis de la etapa analógica en el dominio de la frecuencia determinando las condiciones óptimas de funcionamiento. Las pruebas de campo del equipo se llevaron a cabo en las sierras de Villa Alpina, Córdoba, Argentina. Teniendo en cuenta que el sistema fue diseñado para la medición y estudio de fenómenos electromagnéticos naturales en la banda ELF, se han logrado detectar con éxito las primeras seis resonancias de Schumann.

Palabras clave: antena de lazo; ELF; campos magnéticos; sistema de adquisición; resonancias de Schumann.

\section{INTRODUCTION}

Electromagnetic Fields are present on the Earth surface, due to natural and artificial emissions. The magnetic field of Earth is not only static, but also includes very slow time variations: secular, annual, 27 days, diurnal and substorm magnetic bay type variations at very low frequency. The geomagnetic pulsations phenomena, take place from $1 \mathrm{mHz}$ to $1 \mathrm{~Hz}$. During the past few decades, a remarkable increase in the quality and quantity of electromagnetic data recorded before and during eruptions and earthquakes [1] evidence that seismic movements are preceded by anomalous electromagnetic signals. The Electromagnetic signals as earthquake precursors have been discussed in many publications and are also studied in our research project [2], [3]. Aspects of tectonomagnetism, volcanomagnetism and tectonoelectricity, concentrate on various parts of the electromagnetic spectrum from radio frequencies (RF) to submicrohertz frequencies [1]. Other electromagnetic phenomena in the ULF/ELF band are originated by particles impinging on the magnetosphere causing electromagnetic emissions that propagate inside the magnetosphere cavity [4]. Lightning flashes are the main source of energy for the electromagnetic background inside the ionospheric cavity. Starting from the lower band ELF (few $\mathrm{Hz}$ ) up to VHF (hundreds $\mathrm{MHz}$ ) the noise is originates mainly from the energy radiated by lightning strokes. The main relevant phenomena in the ELF lower band are the Schumann Resonances (SR) [4]. The Earth and the ionospheric layers appear as perfect conductors having air in between, forming an Earth-ionosphere cavity, in which electromagnetic radiation is trapped. Lightning strikes within the troposphere radiate energy into this system and the waves travel around the Earth. In the case of constructive interference, Earth-ionosphere cavity resonances are excited [4]. The SR oscillation detection is a complex procedure which requires customized and high-quality measurement systems. The detection of SR employs the limited energy generated and dissipated by the global lighting activity. The magnetic field amplitudes received are about few tenths of pico Tesla. [5]. The most important naturally occurring VLF signal is the whistler. A whistler is created from a lightning stroke that passes first to the ionosphere and then to the magnetosphere above. These particles are then guided along the Earth's magnetic field, returning to ground to the opposite hemisphere [2].

The study of these very low frequencies electromagnetic phenomena mentioned above, has motivated the construction of a specific acquisition system for this electromagnetic spectrum range. The main objective of this work is focused 
on the characterization of a high-sensitivity antenna used as a sensor for magnetic waves in the ELF band and the system performance as a whole. In the section II a circuit model for the used antenna is proposed. The section III describes the main system specifications, including the antenna, a high-gain amplifier and the digitizing system. Then, the section IV shows the results obtained in the antenna model parameters characterization, the complete system frequency response and the Schumann resonances measured in filed test. Finally, in sections V and VI the general conclusions and some goals that are expected to be carried out in the near future are mentioned.

\section{Antenna Modeling}

\section{A. Proposed design}

For the applications of interest mentioned in this work, the design, implementation and modeling of a high inductance loop antenna with magnetic core is proposed. The chosen design diagram can be seen in the Fig. 1. This antenna consists of eight commercial copper windings with a large number of turns each, connected in series around a high permeability core. This configuration was chosen to have a simpler and more practical assembly. On the other hand, a plastic cover was also incorporated for protection. In later sections more details and characteristics will be seen.

\section{B. Equivalent Circuit Model}

The proposed antenna is essentially an inductor, which can be represented as an ideal inductance $L$. Thus, the simple model for impedance of the antenna results $Z(\omega)=$ $j \omega L$. However, a series resistance $R_{s}$ must be included due to wire losses, obtaining $Z(\omega)=j \omega L+R_{s}$. On the other hand, due to the high inductance for this antenna design, a considerably low resonant frequency occurs, therefore capacitive effect must also be taken into account. Then, an improvement of the model contemplates an equivalent parasitic capacity $C$ which is caused by the copper winding. In this case would be $Z(\omega)=\left(j \omega L+R_{s}\right) / /(j \omega C)^{-1}$. Finally, others losses represented as an equivalent resistance in parallel $R_{p}$ to the whole assembly can also be considered, that is $Z(\omega)=\left(j \omega L+R_{s}\right) / /(j \omega C)^{-1} / / R_{p}$. In this way, in Fig. 2 the definitive circuit model proposed for the loop antenna with a magnetic core is shown. Likewise, the total impedance can also be rewritten as a function of their resistive and reactive parts: $Z(\omega)=R(\omega)+j X(\omega)$. In Equations 1 and 2, both parts are expressed in terms of all electrical parameters and the angular frequency.

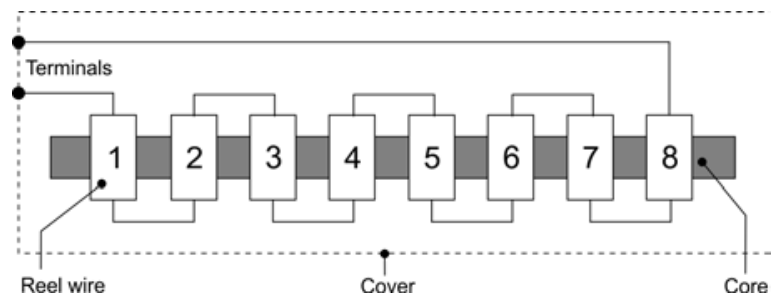

Fig. 1. Proposed design for the loop antenna.

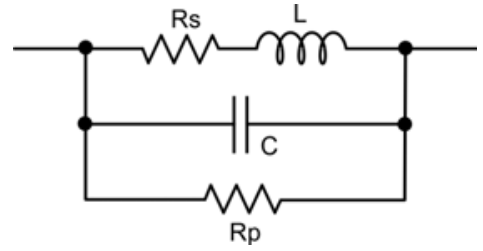

Fig. 2. Equivalent electric circuit of the receiver antenna: ideal inductance $L$; series resistance $R_{s}$; parasitic capacity $C$; parallel loss resistance $R_{p}$.

$$
R(\omega)=\frac{\left(R_{s}+\frac{R_{s}^{2}}{R_{p}}+\omega^{2} \frac{L^{2}}{R_{p}}\right)}{\left(1-\omega^{2} L C+\frac{R_{s}}{R_{p}}\right)^{2}+\left(\omega \frac{L}{R_{p}}+\omega R_{s} C\right)^{2}}
$$

$$
X(\omega)=\frac{\left(\omega L-\omega^{3} L^{2} C-\omega R_{s}^{2} C\right)}{\left(1-\omega^{2} L C+\frac{R_{s}}{R_{p}}\right)^{2}+\left(\omega \frac{L}{R_{p}}+\omega R_{s} C\right)^{2}}
$$

\section{MATERIALS AND SPECIFICATIONS}

This section describes some characteristics and specifications for different components and main stages for a low frequency magnetic field measurement system. In Fig. 3 the sensing system simplified scheme can be seen. It includes the following stages: loop antenna, high gain amplifier + filter, and digital acquisition. These are briefly described below.

\section{A. Antenna Specifications}

An iron core loop antenna with a square section and a large number of wire turns has been built. The antenna is made up of 8 reels of copper wire, as can be seen in Fig. 1, with around 12000 turns each, obtaining a total 96000 turns. In Table I its main design parameters are detailed. Likewise, in Fig. 4 a picture with different parts of the built antenna can be seen. A feature to highlight is the huge number of turns and relatively high core permeability, since this implies a very high inductance which improve its sensitivity for detecting very weak magnetic field signals at very low frequencies.

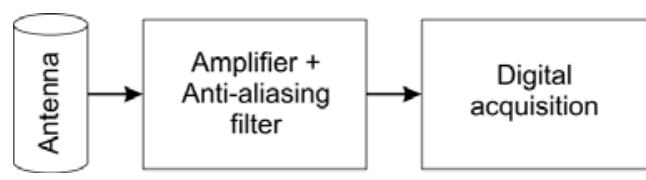

Fig. 3. Stages of the magnetic field measurement system.

TABLE I

LOOP ANTENNA SPECIFICATIONS

\begin{tabular}{|c|c|}
\hline Parameter & Specification \\
\hline \hline Core section & rectangular \\
\hline Side $(\mathrm{m})$ & 0,0285 \\
\hline Core area $\left(\mathrm{m}^{2}\right)$ & $9 \cdot 10^{-4}$ \\
\hline Wire radius $(\mathrm{mm})$ & 0,12 \\
\hline Wire resistance $(k \Omega)$ & 18,6 \\
\hline Number of turns (total) & 96000 \\
\hline$\mu_{r}$ (approx.) & 35 \\
\hline Resonant frequency $(\mathrm{Hz})$ & 200 \\
\hline
\end{tabular}


(a)

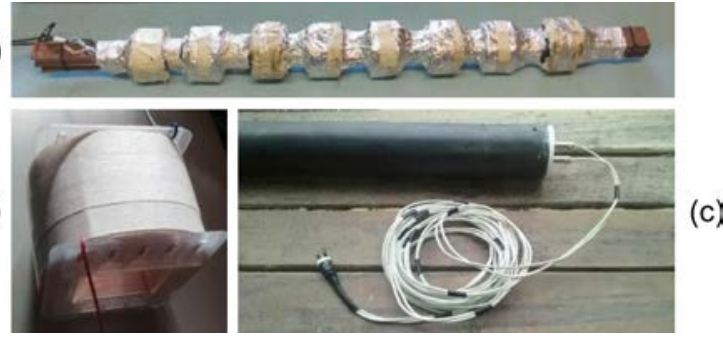

Fig. 4. Loop antenna (high sensitivity). (a) eight reels on core; (b) a single reel; (c) cover protection and antenna connector.

\section{B. High Gain Amplifier and Anti-aliasing Filter}

The voltage induced in the coil (close to micro-volt), is amplified by a high gain amplifier (Minimum Open Loop Amplifier, MOLA) [6] of up to $G_{0}[\mathrm{~dB}] \simeq 88 \mathrm{~dB}$, with a very low common mode rejection ratio. An anti-alising filter is also included to mitigate the signals of $50 \mathrm{~Hz}$ interference due to the public electric service and other higher frequency components. It should be noted that in Villa Alpina the closest public power grid is $8 \mathrm{~km}$ away, which reduces the presence of this type of interference [6]. Even so, the equipment grounding scheme was carefully planed, with all ground references connected to the metal chassis of the testing equipment. All the system was powered by a $12 \mathrm{~V} /$ $75 \mathrm{Ah}$ battery and $\pm 9 \mathrm{~V}$ voltage regulators. The amplifier input resistance has been set at $R_{i n}=39 \mathrm{k} \Omega$ and the output resistance at $R_{o}=1,2 \mathrm{k} \Omega$. For the anti-aliasing filter two capacitors were connected in parallel $4,7 \mu \mathrm{F} / / 470 \mathrm{nF}$ to the amplifier output. This result in a total capacity $C_{o} \simeq$ $5,17 \mu \mathrm{F}$, forming a simple first order low pass filter, whose theoretical cut-off frequency is $f_{c}=\left(2 \pi R_{o} C_{o}\right)^{-1} \simeq 25$ $\mathrm{Hz}$. Later, a more detailed analysis about this aspect will be developed.

\section{Data Acquisition System}

At the amplifier output, the amplified signal is digitized by an analog to digital converter (ADC) with 12-bit resolution and $100 \mathrm{~Hz}$ sampling rate. The input resistance $R_{D}$ for this ADC is considered much higher than amplifier resistance output, that is $R_{D}>>R_{o}$. The acquired data are encoded and stored in an 8 GB micro-SD memory, storing one minute long data for each file, also including additional information as date, time, and other variables detected. For this digital part, a 32-bit microcontroller cortex-M3 LPC1769 was used. All parts of the measurement system has been installed inside a metal cabinet located inside a waterproof plastic enclosure.

\section{Methods And EXPERIMEnTAL Results}

\section{A. Antenna model estimation}

The electrical circuit of the loop antenna has been measured by mean of a LCR meter for frequencies between 12 $\mathrm{Hz}$ and $187 \mathrm{~Hz}$. For measuring lower frequencies $(<12$ $\mathrm{Hz}$ ) a setup with an Oscilloscope and a function generator was used. All results can be observed in the Table III. The typical inductive behaviour of the loop antenna is depicted in the measurement of the reactance $X(\omega)=\Im m\{Z(\omega)\}$ versus the frequency, as can be observed in the Figure 5. In this graph, the reactance can be approximated as a constant slope line for low frequencies thus noticing its inductive behaviour, but changing this trend when the frequency approaches resonance. As for the real part, $R(\omega)=$ $\Re e\{Z(\omega)\}$, it increases with the frequency due to the Joule effect in a conductor and the losses in the magnetic core, as can be seen in Fig. 6.

The estimated curves for the antenna model (dotted lines) are displayed using the best-fitting model to measured data. Parameters values for this model were determined in different ways. The series resistance $R_{s}=18,6 \mathrm{k} \Omega$ was measured directly at DC. The inductance was deduced by computing the slope of the reactance at low frequencies adding a posterior numerical adjustment, resulting in a value close to $L \simeq 800 \mathrm{H}$. Likewise, there is a stray capacitance due to the layers of the loop antenna windings. Therefore, due to stray capacitance and huge inductance, a resonance at unusually low frequencies was observed, resulting $f_{0} \simeq 200$ $\mathrm{Hz}$. Then, for resonance condition $(X(\omega)=0$ in Eq. 2) the stray capacitance is $C=0,79 \mathrm{nF}$ which can be determined by $C=L /\left(\omega_{0}^{2} L^{2}-R_{s}^{2}\right)$. Last, the parallel resistance was estimated using numerical approximations to fit model, obtaining $R_{p}=1,89 \mathrm{M} \Omega$.

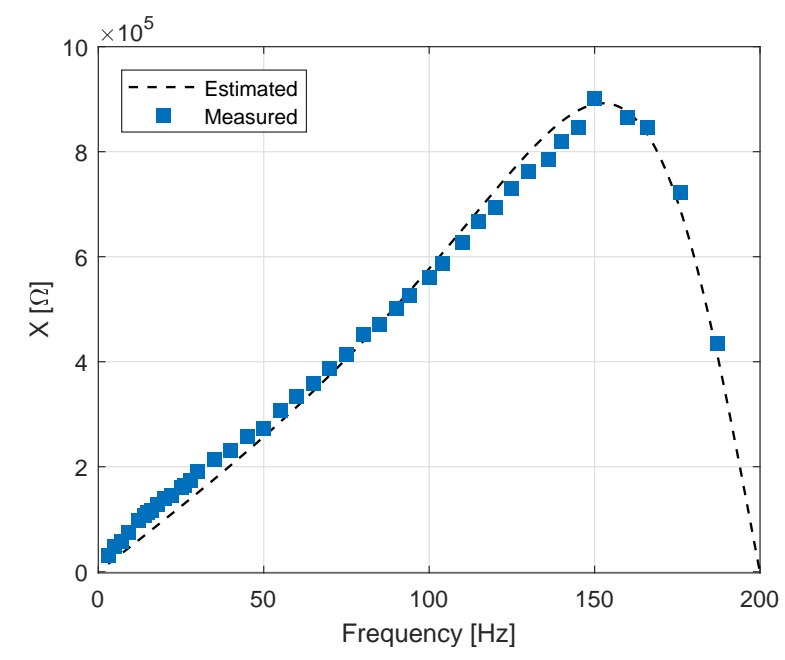

Fig. 5. Imaginary part of the antenna impedance (estimated and measured).

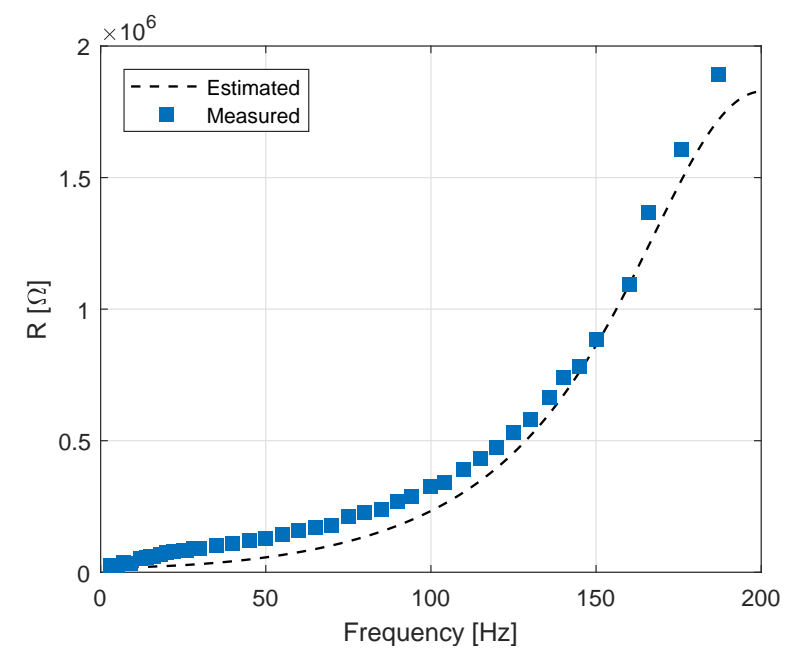

Fig. 6. Real part of the antenna impedance (estimated and measured). 


\section{B. Attenuation at the antenna-amplifier interface}

In Fig. 7 the simplified electrical circuit which describes the connection between antenna and amplifier is shown. The induced voltage $V_{o c}$, which occurs when a magnetic field is present, is connected in series with the antenna impedance itself $Z(\omega)$. The resulting effect is a frequency dependent attenuation at the input interface represented by $R_{i n}$. Considering this situation, the Eq. 3 defines the attenuation $A(\omega)$ as the relationship between open circuit voltage $V_{o c}(\omega)$ and input voltage of the amplifier $V_{i n}(\omega)$. Here, the antenna impedance and amplifier input resistance produce a significant loading effect for the useful frequency range $(0-50 \mathrm{~Hz})$ due to the antenna inductance which is unusually high. This could lead to the conclusion that the problem would be solved by increasing the amplifier input resistance. However, as will be seen later, other conclusions can be obtained by doing a more detailed analysis. In Fig. 8 multiple possible curves for the attenuation $|A(\omega)|$ are shown. In solid blue lines attenuations parameterized with different hypothetical values for the input resistance of the amplifier $R_{i n}$ can be seen. Looking at this attenuation in isolation, can be seen that if the input resistance were high (eg. 3.9 $\mathrm{M} \Omega$ ) the attenuation would be low, while for a low resistance (eg. $680 \Omega$ ) the attenuation would be considerably higher around the resonance. There, $|A(\omega)|$ for the true input resistance $R_{i n}=39 \mathrm{k} \Omega$ (in black dotted line) can also be seen. However, a whole analysis that includes the complete analog stage is necessary to reach a better conclusion in which the aliasing effects are also considered.

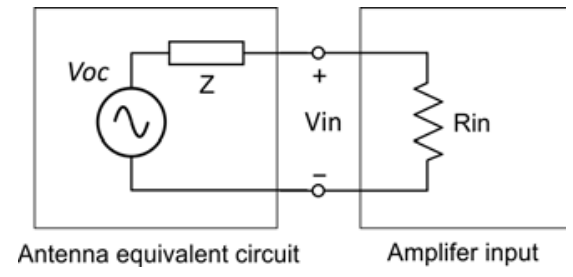

Fig. 7. Connection between the antenna equivalent circuit (induced voltage $V_{o c}(\omega)$ and antenna impedance $\left.Z(\omega)\right)$ and amplifier input resistance $R_{i n}$.

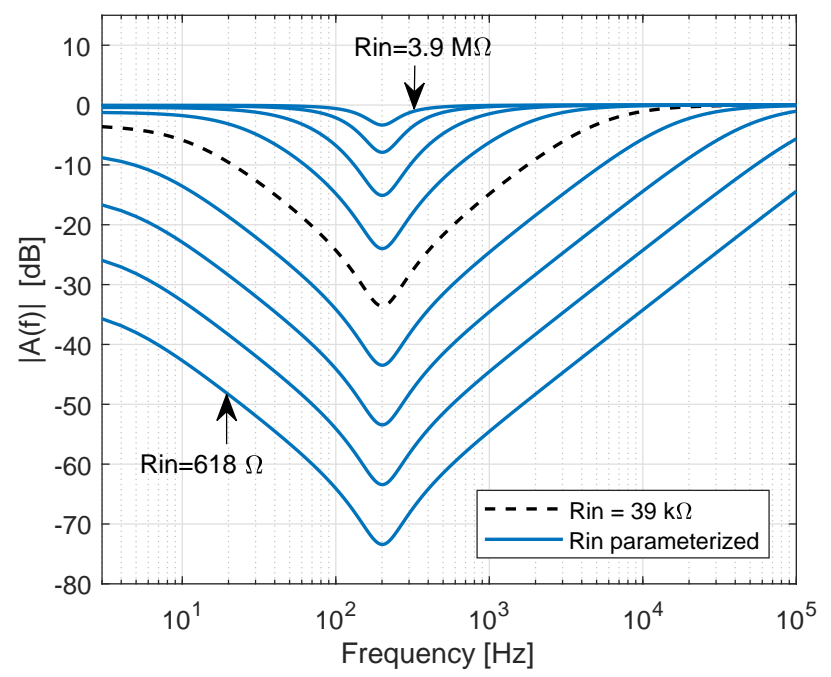

Fig. 8. Input attenuation $|A(f)|(f=2 \pi / \omega)$ parameterized with $R_{i n}$, for hypothetical values (solid blue lines) and true (black dotted lines).

$$
A(\omega)=\frac{V_{i n}(\omega)}{V_{o c}(\omega)}=\frac{R_{i n}}{R_{i n}+Z(\omega)}
$$

\section{Amplifier frequency response}

Considering the equivalent circuit and the voltages indicated in Fig. 9, the transference $V_{\text {out }}(\omega) / V_{\text {in }}(\omega)$ can be defined as expressed in Eq. 4, where $V_{\text {in }}(\omega)$ and $V_{\text {out }}(\omega)$ are the amplifier input and output voltages, respectively. In Fig. 10 the theoretical and measured frequency response magnitude can be seen, showing a cutoff frequency close to $f_{c}=25 \mathrm{~Hz}$ and a maximum gain around $\left.G_{0}\right|_{\mathrm{dB}}=88$ $\mathrm{dB}$.

$$
G(\omega)=\frac{V_{\text {out }}(\omega)}{V_{\text {in }}(\omega)}=\frac{G_{0}}{1+j \omega R_{o} C_{o}}
$$

\section{Total frequency response and induced voltage}

The total transference $T(\omega)$ relates $V_{\text {out }}(\omega)$ with $V_{o c}(\omega)$ and is defined as the product between attenuation and amplification stages. That is $T(\omega)=A(\omega) G(\omega)$, resulting in Eq. 5.

$$
T(\omega)=\frac{V_{\text {out }}(\omega)}{V_{\text {oc }}(\omega)}=\frac{R_{\text {in }}}{R_{\text {in }}+Z(\omega)} \frac{G_{0}}{1+j \omega R_{o} C_{o}}
$$

In analogy to the curves shown for $A(\omega)$, in Fig. 11 the frequency response modulus $|T(\omega)|$ parameterized with $R_{\text {in }}$ is plotted. There, it can be observed the frequency response curve for $R_{\text {in }}=39 \mathrm{k} \Omega$ (dotted black line). Also, it is shown

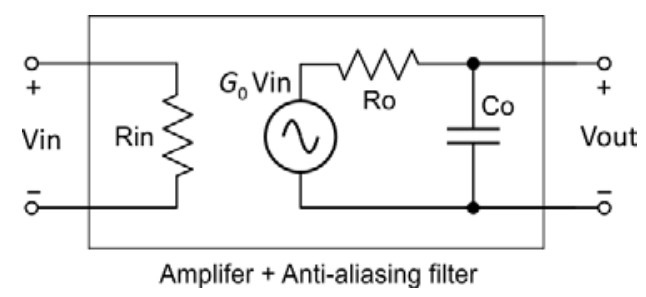

Fig. 9. Amplifier equivalent circuit, where $R_{i n}$ is the input impedance, $G_{0}$ is the maximum gain, $R_{o}$ the output resistance and $C_{o}$ the output capacitance.

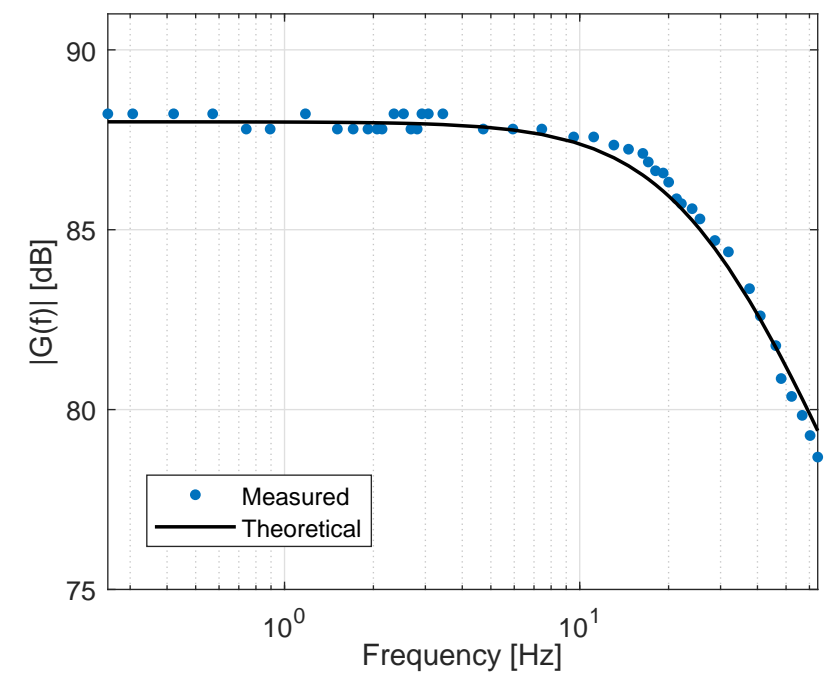

Fig. 10. Amplifier and filter Frequency response $|G(f)|$. 


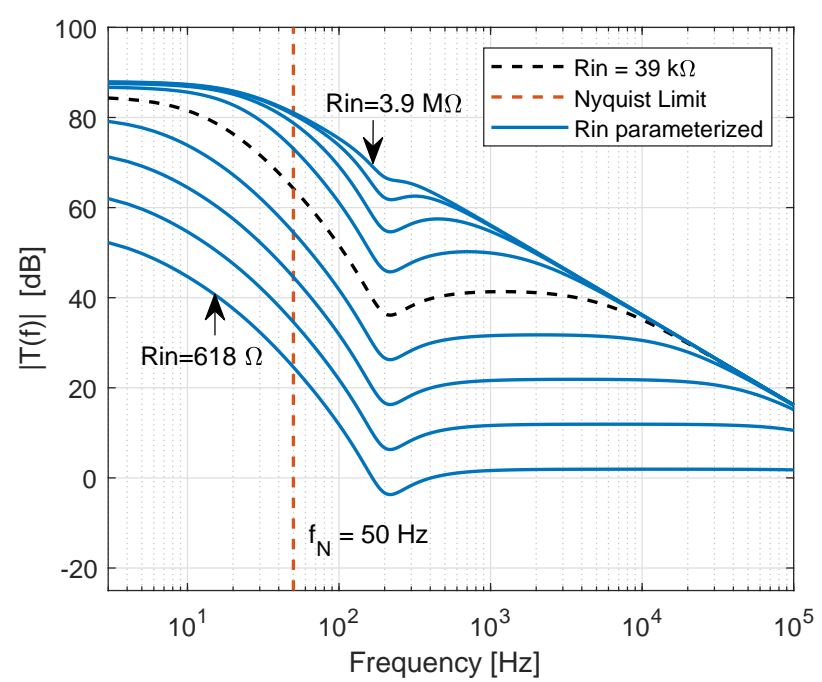

Fig. 11. Total frequency response $|T(f)|$ parameterized with $R_{i n}$, for hypothetical values (solid blue lines) and true (black dotted lines).

that when $R_{\text {in }}$ is big, the frequency response is close to $G(\omega)$, but when $R_{i n}$ is small the behavior is notoriously different.

There are two relevant aspects that can be defined how optimal the measurement system is for the proposed antenna. First, it is important to analyze the gain of the complete system, since the attenuation $A(\omega)$ can significantly reduce the gain provided by the amplifier. This is relevant because the higher the gain, the better the sensitivity of the system. Second, the digital acquisition device has a sampling rate of $f_{s}=100 \mathrm{~Hz}$. This implies a limit on the available spectrum given by the Nyquist frequency, which in this case is $f_{N}=$ $50 \mathrm{~Hz}$. Therefore, to avoid a possible aliasing effect, it is necessary that the frequency behavior of $T(\omega)$ maximizes the energy in the useful band $(0-50 \mathrm{~Hz})$ and minimizes it in the not useful band $(50-\infty \mathrm{Hz})$. From Fig. 11, is important to observe that for low $R_{i n}$ the energy in the not useful range seems to have a better rejection of aliasing but with a large gain reduction, while for high $R_{i n}$, the gain is significantly higher although with a poor behavior against aliasing. To quantify this condition, the ratio between the filter energy in the useful range $\left(0 \leq \omega<\omega_{N}\right)$ and the total energy for all range $(0 \leq \omega<+\infty)$ can be defined as a merit factor. In this way, the Antialiasing Factor (AF) can be expressed as indicated in the equation 6 , where $\omega_{N}=2 \pi 50[\mathrm{rad} / \mathrm{s}]$ is the Nyquist angular frequency.

$$
A F=\frac{\int_{0}^{\omega_{N}}|T(\omega)|^{2} d \omega}{\int_{0}^{+\infty}|T(\omega)|^{2} d \omega}
$$

From this definition, it is possible to analyze the performance of the antialiasing filter by evaluating AF based on the different hypothetical values of $R_{i n}$. In the Fig. 12 this function (expressed in percentage [\%]) can be seen, observing that for both low and high values of $R_{\text {in }}$ the filter behavior is worse for aliasing filtering, while the optimal value that maximizes $\mathrm{AF}$ is given for $R_{\text {in }} \simeq 39 \mathrm{k} \Omega$.

On the other hand, since it is also important to analyze the total system gain, AF can be plotted in function of a refer-
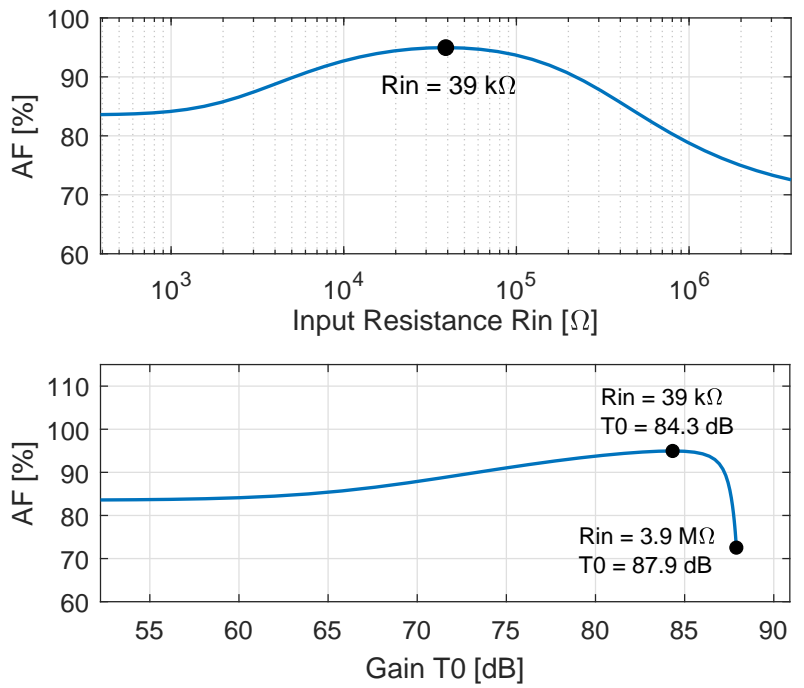

Fig. 12. $\mathrm{AF}[\%]$ as function of input resistance $R_{i n}$ (above) and as function of DC gain $\left.T_{0}\right|_{\mathrm{dB}}$ (below).

ence gain corresponding for each value of $R_{i n}$. In this case, the DC gain $T_{0}=|T(0)|$ has been chosen. Then, according to Fig. 12, AF decreases significantly as $T_{0}$ approaches the maximum possible value, that is $88 \mathrm{~dB}$, which is precisely when $R_{\text {in }} \rightarrow \infty$. It is also noticeable that for the optimal case $\left(R_{\text {in }}=39 \mathrm{k} \Omega\right)$ the gain is $T_{0}=84,3 \mathrm{~dB}$, maintaining a gain relatively close to maximum. However, for a future design, the gain could be enhanced by slightly increasing $R$ for a suboptimal but close to maximum AF value.

Knowing the total analog stage transference, it is possible to obtain the induced voltage $V_{o c}(\omega)$ computing the spectrum of signal $v_{\text {out }}(t)$ which is sampled by the acquisition system. To do this, the spectrum $V_{\text {out }}(\omega)$ can be equalized by applying the inverse of $T(\omega)$ to obtain then $V_{o c}(\omega)$. In Fig. 13, the total transfer (blue solid and black dotted lines) and the equalization function $|T(\omega)|^{-1}$ (black solid line) are plotted. It is important to clarify that for a more general case, this type of equalization can have instability problems if there are zeros located outside left half plane in Laplace's $s$ space, or also noise amplification at high frequencies. Regarding instability, in the presented system there is no such problem since the $T(s)$ only has one pole in the left half plane, so its inverse doesn't have any pole. On the other hand, although there is noise amplification, the useful spectrum for signal is relatively low for $100 \mathrm{~Hz}$ sample rate and the noise amplification can be mitigated by subsequent processing techniques. Then, according to Eq. 7 the compensated spectrum magnitude $\left|V_{o c}(\omega)\right|$ can be determined.

$$
\left|V_{o c}(\omega)\right|=\frac{\left|Z(\omega)+R_{\text {in }}\right| \sqrt{1+\left(\omega R_{o} C_{o}\right)^{2}}}{G_{0} R_{\text {in }}}\left|V_{\text {out }}(\omega)\right|
$$

\section{E. Application: Schumann resonances detection}

Taking into account the objectives of this work, it is important to verify the effectiveness of the proposed antenna in combination with the rest of the system in order to detect very small natural electromagnetic signals. In this 


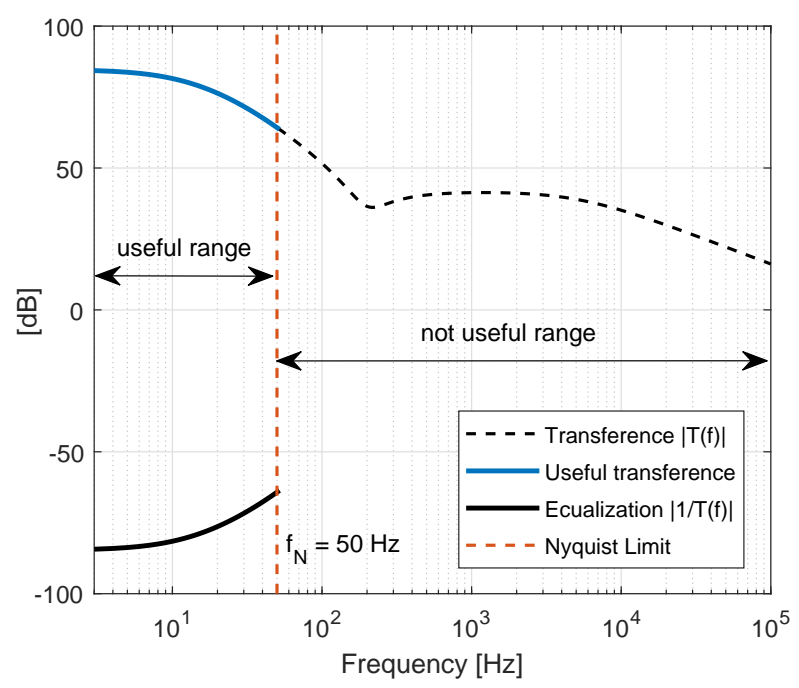

Fig. 13. Total response $|T(f)|$ and $|T(f)|^{-1}$ for $R_{i n}=39 \mathrm{k} \Omega$.

case, the authors have measured the Schumann resonances in the location of Villa Alpina, Córdoba, Argentina. Fig. 14 shows the complete installation of the sensor station on site, which has also been used in other works with similar antennas [7], [6]. This location was chosen due to the very low artificial noise of the power lines radiation and others possible urban interference of higher frequencies [8]. In order to see the spectrum of the amplifier output signal, the power spectral density (PSD), $S_{\text {out }}(\omega)\left[\mathrm{V}^{2} / \mathrm{Hz}\right]$, can be calculated from digitized and stored data. For the better plotted and visibility of the Schumann Resonances peaks, the average PSD can be used, according to some methods seen in [7]. Then, the amplifier output voltage spectrum can be computed as $\left|V_{\text {out }}(\omega)\right|=S_{\text {out }}^{1 / 2}(\omega)[\mathrm{V} / \sqrt{\mathrm{Hz}}]$. This is shown in Fig. 15. There, up to the first six Schumann resonances can be clearly seen. Table II summarizes the mean and deviation of the frequencies estimated using 60 PSDs of one minute long each. Finally, the induced voltage spectrum modulus $\left|V_{o c}(\omega)\right|$ can be calculated by mean of the equation 7 and using the estimation of $\left|V_{\text {out }}(\omega)\right|$, thus obtaining the graph of Fig. 16. An increase in amplitude is noted as the frequency rises, but this characteristic is normal,

TABLE II

SCHUMANN RESONANCES ESTIMATED FROM MEASUREMENTS

\begin{tabular}{|c|c|c|c|c|c|c|}
\hline Parameter & 1st & 2nd & 3rd & 4th & 5th & 6th \\
\hline \hline Mean $[\mathrm{Hz}]$ & 7.84 & 14.23 & 20.67 & 26.67 & 32.53 & 39.83 \\
\hline Deviation $[\mathrm{Hz}]$ & 0.10 & 0.09 & 0.14 & 0.14 & 0.18 & 0.19 \\
\hline
\end{tabular}

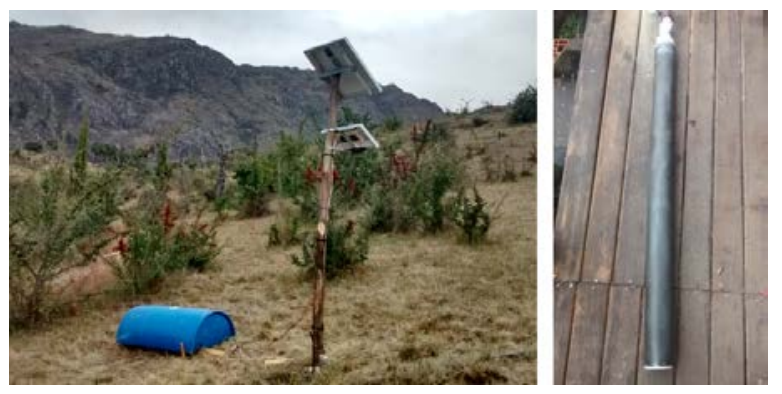

Fig. 14. Installation of the Sensing Station and the antenna in Villa María, Córdoba, Argentina.

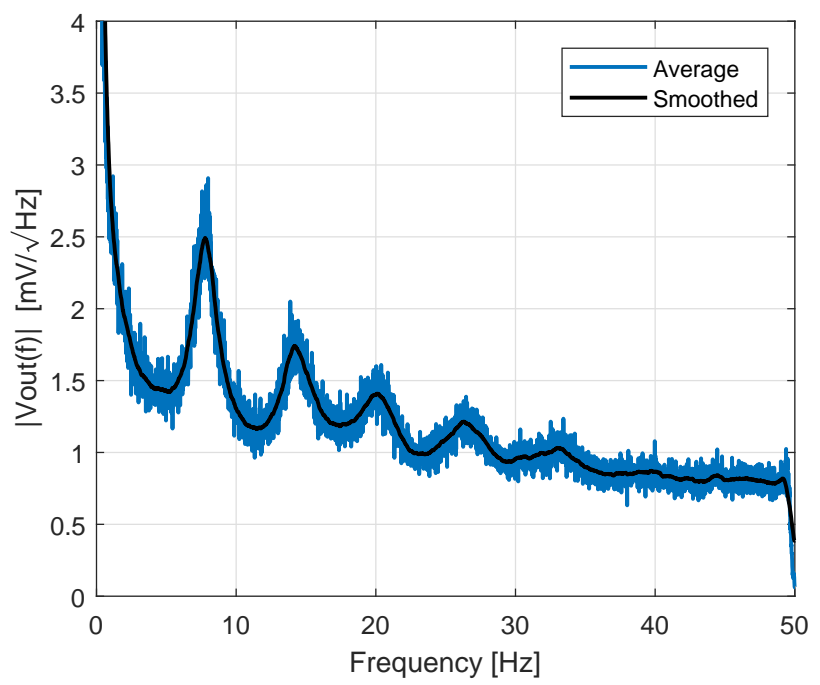

Fig. 15. Average PSD of the voltage measured (60 time windows of one minute each) and smoothed curve. An additional digital filter has also been applied to suppress $50 \mathrm{~Hz}$ components.

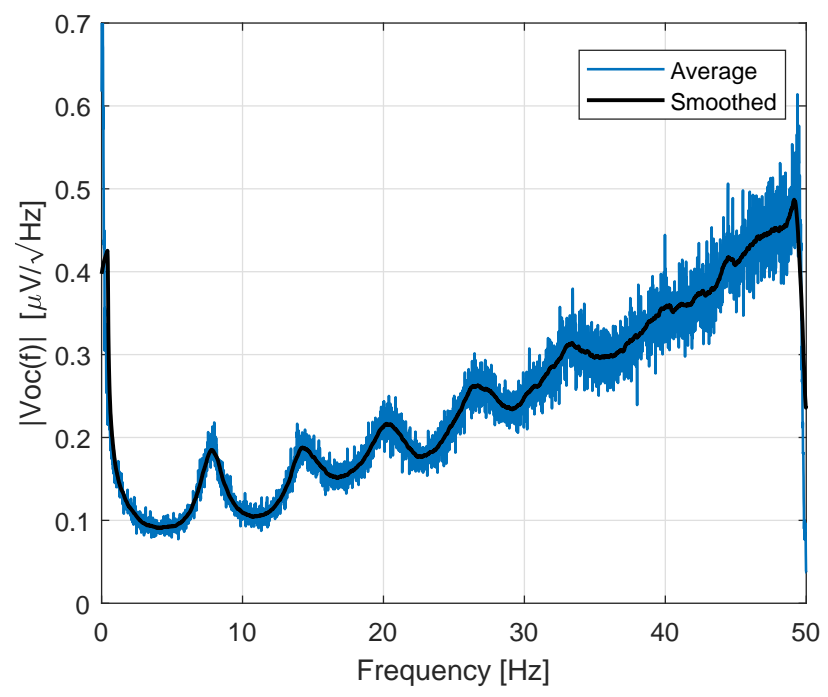

Fig. 16. Average PSD and smoothed curve of the induced voltage estimated after of system equalisation.

as can be seen in similar works such as [9]. Likewise, the last stage in the estimation, which is not part of this work, would be to apply the antenna factor to obtain the spectrum measured in units of magnetic field (tesla), that is $B(\omega)=K(\omega) V_{o c}(\omega)$. The antenna factor $K$ tends to be an inversely proportional to the frequency, so the noise floor would be approximately flat after applying it.

\section{CONCLUSIONS}

In this work we have characterized a loop antenna at very low frequencies to determinate its equivalent impedance model. Also, numeric approximations have been used to estimate other parameters of the model. With these result, a huge inductance $L \simeq 800 \mathrm{H}$ has been verified, which implies a very high sensibility for field sensing. An unusually low frequency of resonance, near to $200 \mathrm{~Hz}$, was observed due to the huge inductance and the parallel stray capacitance. It was found that the proposed antenna together with the rest of the measurement system have a very good performance in the 
TABLE III

MEASUREMENT OF THE LOOP ANTENNA WITH MAGNETIC CORE.

\begin{tabular}{|c|c|c||c|c|c|}
\hline $\mathrm{F}[\mathrm{Hz}]$ & $X \mid k \Omega]$ & $R[k \Omega]$ & $\mathrm{F}[\mathrm{Hz}]$ & $X \mid k \Omega]$ & $R[k \Omega]$ \\
\hline 3 & 30,29 & 26,34 & 70 & 387,04 & 175,93 \\
\hline 5 & 47,25 & 25,4 & 75 & 414,22 & 211,34 \\
\hline 7 & 57,44 & 35,68 & 80 & 452,39 & 226,19 \\
\hline 9 & 75,55 & 33,73 & 85 & 471,05 & 239,11 \\
\hline 12 & 97,26 & 51,46 & 90 & 501,02 & 267,93 \\
\hline 14 & 107,4 & 57,44 & 94 & 525,65 & 287,24 \\
\hline 15 & 113,47 & 59,72 & 100 & 560,46 & 325,85 \\
\hline 16 & 116,62 & 60,42 & 104 & 587,45 & 341,54 \\
\hline 18 & 128,48 & 69,07 & 110 & 627,56 & 389,79 \\
\hline 20 & 140,12 & 73,74 & 115 & 666,21 & 432,6 \\
\hline 22 & 144,31 & 78,86 & 120 & 694,42 & 472,39 \\
\hline 25 & 161,01 & 82,57 & 125 & 730,42 & 529,29 \\
\hline 26 & 164,67 & 83,59 & 130 & 762,01 & 581,68 \\
\hline 28 & 174,7 & 89,59 & 136 & 785,3 & 665,51 \\
\hline 30 & 190,38 & 90,66 & 140 & 819,83 & 738,59 \\
\hline 35 & 213,31 & 101,58 & 145 & 845,47 & 782,84 \\
\hline 40 & 231,72 & 108,79 & 150 & 901,01 & 883,34 \\
\hline 45 & 257,01 & 122,39 & 160 & 864,57 & 1094,39 \\
\hline 50 & 273,32 & 130,15 & 166 & 846,92 & 1366,01 \\
\hline 55 & 307,22 & 143,56 & 176 & 723,22 & 1607,16 \\
\hline 60 & 332,88 & 158,82 & 187 & 434,73 & 1890,15 \\
\hline 65 & 359,4 & 171,47 & - & - & - \\
\hline
\end{tabular}

range of frequencies of interest. This is verified by analyzing the total transfer of the analog measurement stage, observing an optimal behavior for aliasing filtering and guaranteeing a high gain. The resulting portable equipment showed a very high sensitivity, proving that it can detect very weak natural signals at extremely low frequencies. As a particular application case, the first six Schumann resonances have been detected successfully with a great precision.

\section{FUTURE WORKS}

It is expected to measure antenna factor to determinate the magnetic filed $B$ (tesla) from voltage measured. To do this, a measurement bench with Helmholtz coils of a suitable size will be used to generate a known magnetic field as a calibration reference. Likewise, tests will be carried out with other magnetic materials and also assembly three antennas that allow monitoring the three spatial components of the near field.

\section{ACKNOWLEDGMENT}

The authors would like to thank to Edgardo Maffia from Electronica Aplicada Company for the help during experimental process. To Norman Trench for taking care of the installation of equipment and the magnetic field measurements. To Julio Zola and Enrique Zothner for providing a working prototype of the high gain amplifier. To the Universidad de Buenos Aires for the project Grant 20020150100085.

\section{REFERENCES}

[1] M. J. S. JOHNSTON, "Review of electric and magnetic fields accompanying seismic and volcanic activity," Surveys in Geophysics, vol. 18, no. 5 , p. 441-475, 1997.

[2] W. G. Fano, G. Quintana, and R. Alonso, "3D magnetic field measurement at ELF and SLF frequencies," IEEE 2018 GEMCCON, 2018.

[3] G. I. Quintana, R. Alonso, and W. G. Fano, "Loop antenna characterization for ELF and SLF measurements," Elektron, Universidad de Buenos Aires, Argentina., vol. 2, no. 2, p. 95-100, 2018.

[4] C. Bianchi and A. Meloni, "Loop antenna characterization for ELF and SLF measurements," ANNALS OF GEOPHYSICS., vol. 50, no. 3, p. 435-445, 2007.

[5] C. I. Votis, G. Tatsis, V. Christofilakis, S. K. Chronopoulos, P. Kostarakis, V. Tritakis, and C. Repapis, "A new portable ELF Schumann resonance receiver: design and detailed analysis of the antenna and the analog front-end," EURASIP J. Wirel. Commun. Netw., vol. 2018, p. 155, 2018. [Online]. Available: https://doi.org/10.1186/s13638-018-1157-7

[6] N. Trench, R. Alonso, L. M. Carducci, J. Kelly, E. Maffia, E. Zothner, J. Zola, and W. G. Fano, "Sistema de medición remota de campo magnético para señales precursoras sísmicas de muy bajas frecuencias," in Latinmag 2019, Dec 2019. [Online]. Available: https://www.elatinmag.com/aplimetodos-103-trench

[7] L. M. Carducci, R. Alonso, F. Luna, J. Zola, E. Zothner, and W. G. Fano, "Aplicaciones y métodos de medición y procesamiento de señales para la detección de anomalías de campo magnético de muy bajas frecuencias," LATINMAG 2019, 2019.

[8] E. Maffia, V. Trainotti, W. G. Fano, and N. Trench, "Medición de la resonancia de Schumann," Latinmag Letter, vol. 1, no. A02, pp. 1-8, 2011.

[9] G. T. et al, "Design and implementation of a test fixture for ELF Schumann resonance magnetic antenna receiver and magnetic permeability measurements," Electronics, 2020, 9, 171, 2020. 